\title{
Atypical presentation of acute aortic dissection: a
} \section{case report}

\begin{abstract}
We present the clinical case of an 80-year-old woman admitted to the emergency department with jaw pain and acute neurovascular impairment with right arm monoparesis, pulse deficit and pallor of the limb, without chest pain as a cardinal symptom. The history of arterial hypertension, aortic stenosis, and clinical manifestation suggested an acute vascular compromise, for which acute aortic syndrome (AAS) was suspected. Chest $\mathrm{x}$ - rays revealed mediastinal widening; angio-CT scan confirmed type-A aortic dissection affecting the right subclavian artery (thrombosis). The patient passed away after expectant non-surgical management.
\end{abstract}

Keywords: acute aortic dissection, acute aortic syndrome, thrombosis, chest X-rays, vascular affection, anti-impulse therapy
Volume 13 Issue 4 - 2020

\author{
Diego Abreo,' Giovanny Moncayo, ${ }^{2}$ Tatiana \\ Arroyave, ${ }^{3}$ Gabriel Caicedo ${ }^{4}$ \\ 'Department of Emergency Medicine, Hospital Pablo Tobon \\ Uribe, Colombia \\ ${ }^{2}$ Department of Emergency Medicine, Clinica CES, Colombia \\ ${ }^{3}$ Department of General Physician, Hospital Pablo Tobón Uribe, \\ Colombia \\ ${ }^{4}$ Department of Emergency Medicine, Universidad CES, \\ Colombia
}

Correspondence: Diego Abreo, Specialist in Emergency Medicine. Hospital Pablo Tobon Uribe, Colombia, Tel +57 3188370838, Email abreo87@gmail.com

Received: May 13, 2020 | Published: August 28, 2020
Abbreviations: AAD, acute aortic dissection; AAS, acute aortic syndrome; $\mathrm{AD}$, aortic dissection; IRAD, international registry of acute aortic dissection

\section{Introduction}

Acute aortic dissection (AAD) is a vascular emergency with high mortality; prompt detection from emergency services is important based on the likelihood according to risk factors and clinical presentation, which is not always limited to chest pain. The angio$\mathrm{CT}$ scan is of great importance to define the diagnosis and surgical management in most cases. Conservative treatment of AAD, as in this case, has a high mortality rate. This report demonstrates the variety of the presentation of the acute aortic syndrome.

\section{Clinical case}

A 80-year-old female patient with past medical history of arterial hypertension, hypothyroidism, chronic lung disease, hiatal hernia, and moderate aortic stenosis was admitted to the emergency department for one hour of evolution of pain in the submandibular region and the right arm, with loss of strength in this limb, without dyspnea or chest pain. Physical examination revealed hypotension, without altered consciousness or tachycardia, right arm with decreased force, coldness, weak radial pulse, and prolonged capillary refill, adequate femoral pulses, and normal cardiopulmonary auscultation. Acute aortic pathology was suspected; chest X-rays showed mediastinal widening; due to the high probability and clinical suspicion of an acute aortic syndrome (AAS), an angio-CT scan was requested, showing type-A aortic dissection with involvement of the right subclavian artery (thrombosis) and the brachiocephalic trunk. The patient was referred to another institution for assessment by cardiovascular surgery, who analyzed the case and due to the patient's comorbidities, age, and extension of the vascular affection; non-surgical expectant management was decided. She remained in ICU for 24 hours. The next day, the patient presented a convulsive episode, followed by cardiorespiratory arrest. No CPR maneuvers were performed due to previous dissent; the patient died (Figure 1).

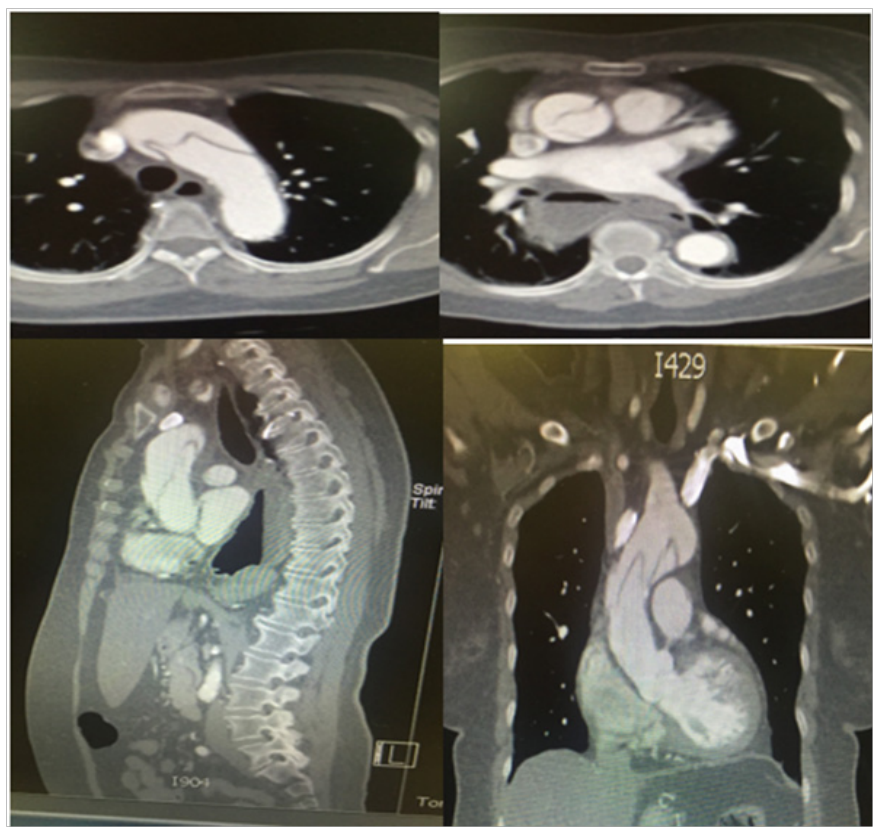

Figure I Angio-CT scan: Type- A aortic dissection that extends to the right brachiocephalic trunk. Thrombosis of the right subclavian artery. Evidence of atherosclerosis, hiatal hernia, simple hepatic cysts, fatty liver, right renal cyst with calcified walls, and simple cyst in the left kidney

\section{Discussion}

Acute aortic syndrome (AAS) encompasses aortic dissection (AD), intramural hematoma and aortic ulcer, the former being the most prevalent, up to $80 \% .{ }^{1,2}$ It may also be due to traumatic causes, constituting the second cause of mortality in about $80 \%$ of the subjects. ${ }^{3,4}$ The dissection can be classified into two groups: Stanford type $\mathrm{A}$ and $\mathrm{B}$, with the first one involving the ascending aorta, and the second one confined to the descending part; and the DeBakey classification with type 1 that involves the entire extension of the aorta, type 2 the ascending portion, and type 3 is limited to the descending 
aorta. ${ }^{5}$ The most known risk factors for the appearance of this condition are atherosclerosis, smoking, dyslipidemia, cardiac surgery, autoimmunity, connective tissue disease, cardiovascular family history, valvulopathy, and in more than $70 \%$, arterial hypertension.

In the IRAD study (International Registry of Acute Aortic Dissection) conducted in 12 reference centers, it was observed that the clinical presentation of patients with $\mathrm{AD}$ is protean, evidencing that chest pain occurs in $72.2 \%$ of patients; ${ }^{6}$ for Spanish surveys such as RESA, this symptom presented in $95.9 \%{ }^{1}$ the sudden onset of symptoms occurred in $95.5 \%$, back pain $53 \%$, abdominal pain $29 \%$, and syncope (14\% in RESA and $9.4 \%$ in IRAD studies). Recent publications describe increased neurological manifestations with respect to previous studies: $17 \%$ are limb motor deficits, being these manifestations more persistent in type-A dissection.

The most frequent clinical findings in the IRAD study were the presence of aortic murmur (31.6\%) and pulse deficit in $15 \%$ of the cases. In the present report, the patient was diagnosed under neurological findings; according to the literature, this type of manifestations correspond to less than $10 \%$ of the cases, including: syncope, coma, dysphonia, spinal cord injury or cerebrovascular accident, ${ }^{6}$ most of these are transient (in $15-40 \%$ of the cases) and explained by four physiopathological mechanisms: poor perfusion, hypotension, distal thromboembolism, and compression of peripheral nerves. ${ }^{5}$ The ischemic neuropathy of the limbs secondary to poor perfusion is mainly due to subclavian and femoral involvement in up to $10 \%{ }^{5}$ For European publications, the neurological deficit occurs in the same proportion but includes $8 \%$ of patients who present with pain in one limb. ${ }^{1,5}$

The diagnosis of the SAA proposed by AHA in $2010^{7}$ is based initially on the pre-test probability according to the risk factors, the characteristics of the pain, and the clinical examination; the presence of two or more of these factors constitute a high probability, being necessary an imaging request; they initially recommend transthoracic echocardiography followed by an angio-CT scan. Patients with low probability require additional studies such as chest X-rays and D-dimer. ${ }^{5,7}$ X-rays may be normal in $21 \%$ of cases. ${ }^{6}$ Angio-CT scan is the gold standard for diagnosis. ${ }^{5}$

The importance of knowing this entity lies in the variability of the presentation along with the morbidity and mortality that it comprises; type-A dissections are considered the most common according to the IRAD survey, corresponding to $62 \%$; and of these, those that undergo surgery have an in-hospital mortality of $26 \%$ against $58 \%$ in those that receive medical treatment (due to comorbidities, age or rejection of surgical management). Type-B dissection has a mortality rate of $31 \%$ in surgical management versus $10 \%$ under medical management. ${ }^{6}$ The early recognition of this pathology depends on the clinical suspicion of the physicians in the emergency department; there are reports that demonstrate that this pathology only occurs in up to $15 \%$ of patients as the first diagnostic impression; ${ }^{8}$ in $85 \%$ of the cases, the diagnosis is delayed. Other publications report that up to $40 \%$ of patients are not diagnosed with the initial symptom. ${ }^{2,9,10}$ Surgical complications include spinal cord injury $(6.8 \%)$, stroke $(9 \%)$, mesenteric ischemia $(4.9 \%)$, and acute kidney injury (19\%). Endovascular management is another invasive method that is used for type-B dissection and carries similar surgical risks. ${ }^{11}$

Medical management is based on anti-impulse therapy consisting of management in high-dependency units, pain control, and mainly stabilization of heart rate and blood pressure, seeking for goals of 60 to 80 beats per minute and $100-120 \mathrm{mmHg}$ of systolic blood pressure. ${ }^{6}$ The drugs of choice for analgesia are opioids and betablockers such as esmolol or labetalol to control blood pressure and heart rate.

\section{Conclusion}

These atypical manifestations of the case are presented to inform the key professionals for the diagnosis of this cardiovascular emergency, whose manifestation is not always the usual; and, in this way, to be able to establish prompt management, impacting on the morbidity and mortality of the patients.

\section{Acknowledgments}

None.

\section{Conflicts of interest}

Author declares that there are no conflicts of interest.

\section{Funding}

None.

\section{References}

1. Evangelista A, Padilla F, Lopez-Ayerbe J, et al. Spanish Acute Aortic Syndrome Study (RESA). Better Diagnosis Is Not Reflected in Reduced Mortality. Rev Eso Cardiol. 2009;62(3):255-262.

2. Lech C, Swaminathan A. Abdominal aortic emergencies. Emerg Med Clin N Am. 2017;35(4):847-867.

3. Strayer R. Thoracic aortic syndromes. Emerg Med NAm. 2017;35(4):713725 .

4. Trust M, Teixeira P. Blunt trauma of the aorta, Current Guidelines. Cardiol Clin. 2017;35(3):441-451.

5. Erbel R, Aboyans V, Borleau C, et al. Guía ESC 2014 sobre el diagnóstico y tratamiento de la patología de la aorta. Rev Esp Cardiol. 2015;68(3):242.e1-e69.

6. Hagan P, Nienaber C, Isselbacher E, et al. The international Registry of Acute Aortic Dissection (IRAD). New insights into an old disease. JAMA. 2000;283(7):897-903.

7. Hiratzka L, Bakris G, Beckman J, et al. ACCF/AHA/AATS/ACR/ ASA/SCA/SCAI/SIR/STS/SVM Guidelines for the Diagnosis and Management of Patients With Thoracic Aortic Disease: Executive Summary. Circulation. 2010;121(13):e266-e369.

8. Mészáros L, Mórocz J, Szlávi J, et al. Epidemiology and clinicopathology of aortic dissection. Chest. 2000;117(5):1271-1278.

9. Rylski B, Suedkamp M, Beyersdorf F, et al. Outcome after surgery for acute aortic dissection type $\mathrm{A}$ in patients over 70 years: data analysis from the German Registry for Acute Aortic Dissection Type A (GERAADA). Eur J Cardiothorac Surg. 2011;40(2):435-440.

10. Fattori R, Cao P, De Rango P, et al. Interdisciplinary expert consensus document on management of type B aortic dissection. J Am Coll Cardiol. 2013;61(16):1661-1678.

11. Higa C, Guetta J, Borraci R, et al. Multicenter registry of acute aortic dissection. RADAR study. Preliminary results. Rev Argent Cardiol. 2009;77:354-360. 